\title{
Genetics of osteoarthritis: early developmental clues to an old disease
}

\author{
Eline Slagboom and Ingrid Meulenbelt
}

About 15 years ago, it was considered questionable whether genetic research into a so-called wearand-tear disease, such as osteoarthritis (OA), would provide any useful insight. Since then, a number of middle-aged patient populations have been investigated in the quest for OA susceptibility genes. The best genetic studies have revealed that $\mathrm{OA}$ is a degenerative disease seen in people of advanced age, which involves cartilage maintenance functions, determinants of bone composition and an intriguing developmental component.

$\mathrm{OA}$ is the most common chronic joint disease in the elderly. Radiographic signs of OA correspond with the aging process of joints and, in approximately $50 \%$ of affected individuals, are associated with clinical manifestations, such as chronic pain, joint stiffness and long-term disability. Current treatment is mainly limited to drug-based pain relief and total joint replacement. Trauma, obesity, age and genetic factors are the culprits of OA pathogenesis. Genetic predisposition is relevant for different definitions of OA, based on the affected joint site and on progression of the disease.

Despite the heterogeneity in phenotype definitions applied in different study populations, consistently confirmed OA susceptibility genes have been identified in the Wnt, transforming growth factor- $\beta$ and thyroid signaling pathways (Bos S et al. [2008] Curr Opin Rheumatol 20: 553-559). These loci, including those for the genes frizzled-related protein $\beta$ (FRZB), growth differentiation factor 5 (GDF5), deiodinase, iodothyronine, type II (DIO2) and double von Willebrand factor A domains (DVWA), are involved in skeletal morphogenesis. FRZB is expressed in the proliferative zone of the growth plate, where it antagonises the Wnt signaling pathway. GDF5 expression has a role in the early development of joints in embryonic tissues and is expressed within the synovial

\section{...the next \\ question to \\ be addressed \\ is how these \\ genes influence the onset of OA at a later stage in life}

\section{E Slagboom is a Professor and I Meulenbelt is an Associate Professor at the Molecular Epidemiology Section, Leiden University Medical Centre, Leiden, The Netherlands.}

\section{Competing interests} The authors declared no competing interests.

www.nature.com/clinicalpractice doi:10.1038/ncprheum0935 joint tissues throughout life, where it promotes bone morphogenic cell signaling. The DIO2 gene encodes an enzyme that regulates intracellular bioavailability of active thyroid hormone $\mathrm{T}_{3}$. In the growth plate, $T_{3}$ initiates the terminal differentiation of hypertrophic chondrocytes, which is important for the subsequent formation of bone. DVWA physically interacts with $\beta$-tubulin, which also regulates chondrocyte differentiation in the growth plate. As yet, only an FRZB knockout mouse model has been investigated for OA. This mouse has a normal skeletal morphogenesis, but increased bone mineral density and stiffness. The mice develop OA-like phenotypes, but only following mechanical challenges (Lories RJ et al. [2007] Arthritis Rheum 56: 4095-4103).

Now that investigations into the genetics of OA have indicated a role for genes in the endochondral ossification during early skeletal development, the next question to be addressed is how these genes influence the onset of $\mathrm{OA}$ at a later stage in life. One hypothesis is that early suboptimal skeletal morphogenesis increases OA susceptibility at middle age. Another possibility is that the gene variants contribute to chondrocyte dedifferentiation later in life. In mature, healthy articular cartilage, chondrocytes remain in maturational arrest, showing no proliferation and low metabolic activity, whereas in OA, they dedifferentiate into a metabolically active, hypertrophic state. In this dedifferentiated state, which other tissues also seem to undergo in aging, OA chondrocytes share a fair amount of their expressed genes with those in the terminal layer of the growth plate. These considerations justify future research into other potential OA susceptibility genes involved in chondrogenic differentiation during endochondral ossification. Research focusing on factors that enhance the phenotypic stability of the aging articular chondrocyte could, therefore, pave the way for new OA therapeutics. 